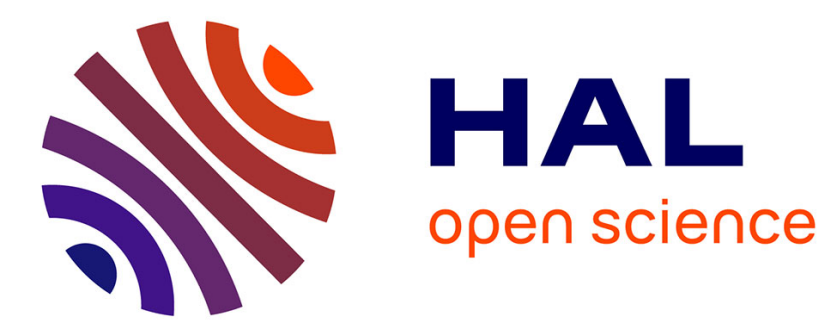

\title{
Impact of defect tolerance techniques on the criticality of a SRAM-based Mesh of Cluster FPGA
}

Adrien Blanchardon, Roselyne Chotin-Avot, Habib Mehrez, Emna Amouri

\section{To cite this version:}

Adrien Blanchardon, Roselyne Chotin-Avot, Habib Mehrez, Emna Amouri. Impact of defect tolerance techniques on the criticality of a SRAM-based Mesh of Cluster FPGA. ReConFig 2014 - International Conference on ReConFigurable Computing and FPGAs, Dec 2014, Cancun, Mexico. pp.1-6, 10.1109/ReConFig.2014.7032508 . hal-01162066

\section{HAL Id: hal-01162066 \\ https: / hal.sorbonne-universite.fr/hal-01162066}

Submitted on 9 Jun 2015

HAL is a multi-disciplinary open access archive for the deposit and dissemination of scientific research documents, whether they are published or not. The documents may come from teaching and research institutions in France or abroad, or from public or private research centers.
L'archive ouverte pluridisciplinaire HAL, est destinée au dépôt et à la diffusion de documents scientifiques de niveau recherche, publiés ou non, émanant des établissements d'enseignement et de recherche français ou étrangers, des laboratoires publics ou privés. 


\title{
Impact of defect tolerance techniques on the criticality of a SRAM-based Mesh of Cluster FPGA
}

\author{
Adrien Blanchardon ${ }^{1,2}$, Roselyne Chotin-Avot ${ }^{1,2}$, Habib Mehrez ${ }^{1,2}$, Emna Amouri ${ }^{1,2}$ \\ 1- Sorbonne Universités, UPMC Univ Paris 06, UMR 7606, LIP6, F-75005, Paris, France \\ 2- CNRS, UMR 7606, LIP6, F-75005, Paris, France \\ Email: adrien.blanchardon@lip6.fr
}

\begin{abstract}
As device sizes shrink, circuits are increasingly prone to manufacturing defects. One of the future challenges is to find a way to use a maximum of defected manufactured circuits. One possible approach to this growing problem is to add redundancy to propose defect-tolerant architectures. But, hardware redundancy increases area. In this paper, we propose a method to determine the most critical elements in a SRAMbased Mesh of Clusters FPGA and different strategies to locally insert hardware redundancy. Depending on the criticality, using defect tolerance, area and timing metrics, five different strategies are evaluated on the Mesh of Clusters architecture. We show that using these techniques on a Mesh of Clusters architecture permits to tolerate 4 times more defects than classic hardware redundancy techniques applied on industrial mesh FPGA. With local strategies, we obtain a best trade off between the number of defects bypassed (37.95\%), the FPGA area overhead $(21.84 \%)$ and the critical path delay increase $(9.65 \%)$.
\end{abstract}

\section{INTRODUCTION}

The increase of integration density according to Moore's law is being slowed due to economic and physical limits. As manufacturing yield decreases, one of the future challenges is to find a way to use a maximum of manufactured circuits tolerating physical defects all over the chip [1] [2]. Compared to ASICs, FPGAs have attained a central focus due to their ability to integrate more complex applications, their flexibility and good performance. Considering that FPGAs based on nanotechnology may have a defect rate of 20\% [3] [4], propose techniques to avoid these defects is necessary. Several studies examined the effect of redundancy techniques on the performance of FPGAs. These techniques can be classified into software-based and hardware-based techniques. Softwarebased techniques avoid defective resources using configuration tools [5] [6] [7] [8]. However, their efficiency relies on the ability of the tools to take into account the defects and the number of unused resources. Hardware-based techniques use spared resources in the architecture. Correct a defect is to bypass the defective element by the added one [9] [10] [11]. Hardware-based techniques can be implemented in FPGAs to increase their reliability. The studies in [12] and [13] showed that using hardware redundancy on the top level of the FPGA requires more area to tolerate the same number of defects than a hardware redundancy applied inside local interconnect. As theses techniques increase significantly the FPGA area, it is therefore necessary to apply a local redundancy inside the FPGA depending on the criticality of each block. The focus of this paper is to present a method to determine the criticality of each block and to apply different local hardware-based techniques to prevent defects without much decrease performance. The remainder of the paper is organized as follows. Section II describes the architecture of the FPGA under study. Section III presents different defect-tolerant schemes applied at different levels in the FPGA. Section IV details the proposed method to determine the criticality of each block. Simulation results are presented in section V. Finally, section VI concludes this paper.

\section{FPGA ARCHITECTURE}

In FPGAs, the interconnect takes up $90 \%$ of the total area and is responsible of a large part of the circuit delay [14] [15]. To reduce these costs, we propose in [14] a Mesh of Clusters architecture (Fig.1). With this kind of architecture based on clustering, the total area can be reduced by $42 \%$ compared to the VPR Style clustered Mesh.

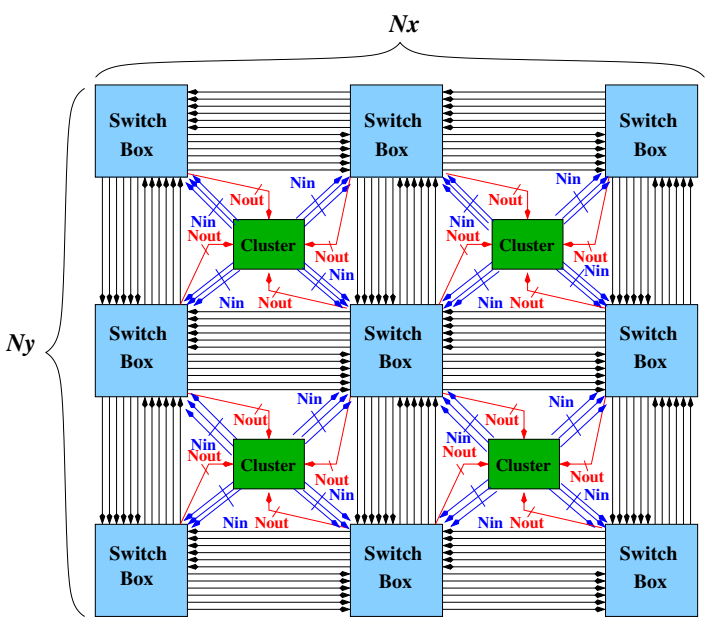

Fig. 1: Mesh of Cluster architecture

This architecture contains clusters placed into a regular 2dimensional grid. Each cluster (Fig.2) contains Configurable Logic Blocks(CLBs) and Mini Switch Box (MSB) to connect them. Each Downward Mini Switch Box (DMSB) is used to connect all cluster's inputs to the CLB's inputs while the Upward Mini Switch Box (UMSB) connects all CLB's outputs to the cluster's outputs. All UMSBs outputs are also connected to the DMSBs as feedbacks to reuse CLB's outputs and 
connect CLBs together. This cluster architecture is generic, the number of inputs/outputs of a cluster is chosen in agreement with the Rent parameter [16].

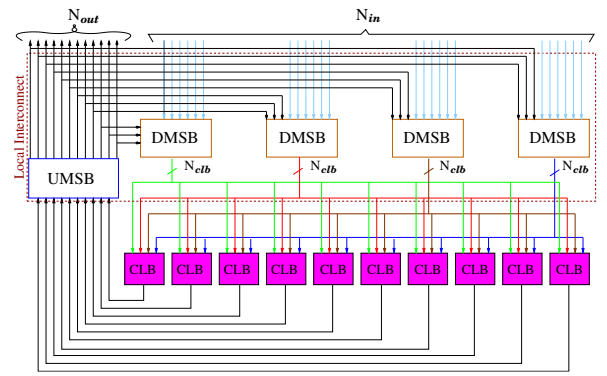

Fig. 2: Initial cluster

A Switch Box (Sbox)(Fig.3) is composed of UMSBs and DMSBs placed on 2 hierarchical levels (DMSB1 and DMSB2). Each DMSB2 is used to connect Sboxs together and to connect all Sbox's inputs on DMSB1. Furthermore, DMSB1 has outputs connected to the adjacent clusters (Fig.1). Finally, UMSB connects adjacent cluster outputs to the DMSB1 and DMSB2. Thus, each cluster is connected to adjacent cluster and to adjacent Sbox. This Sbox architecture is generic, each MSB is composed of multiplexers $N: 1$ (Mux) and each multiplexer is composed of multiplexers 2:1 (mux2). There are as many multiplexers (Mux) as MSB's outputs.

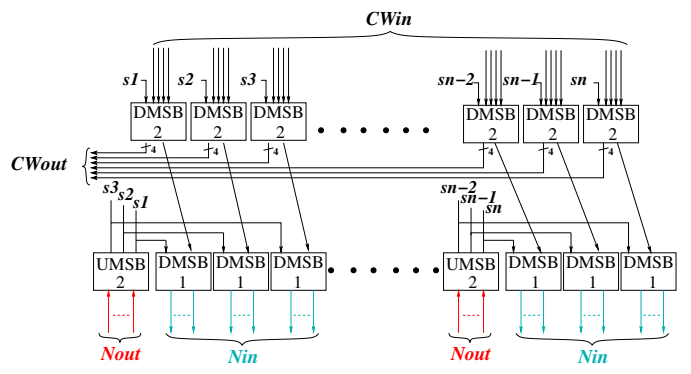

Fig. 3: Initial Sbox

\section{HARDENING THE FPGA}

The next paragraphs review how to adapt for the Sbox the different hardware-based techniques introduced in [17] which are based on hardware redundancy on MSB. To bypass a defect, its location is needed to configure the application. This is done with a map of all defected multiplexers after test and diagnosis [11].

\section{A. Hardware redundancy in Mesh of Clusters architecture}

In a Mesh of Clusters architecture, if a signal is routed by a defective multiplexer in a MSB, the connection between this signal and its corresponding target is not possible. The presented techniques permit to solve this problem. To adapt the Fine Grain Redundancy technique (FGR) [12] for the Sbox of a Mesh of Clusters architecture, four levels of multiplexers (Mux) are added around all the MSB of the Sbox. As the FGR technique is useless if the nearest multiplexer is already used by another input, the Improved Fine Grain Redundancy (IFGR) was proposed in [18] to avoid this kind of conflicts. This solution doubles the number of outputs per multiplexer that allows to use the second output in case of defect on the other one. Then, several techniques were proposed in [17] to improve defect tolerance of the cluster : Adapted Fine Grain redundancy (AFGR), Distributed Feedbacks (DF) [11] and Upward Redundant Multiplexer (URM). These techniques can also be applied on the Sbox (Fig.4).

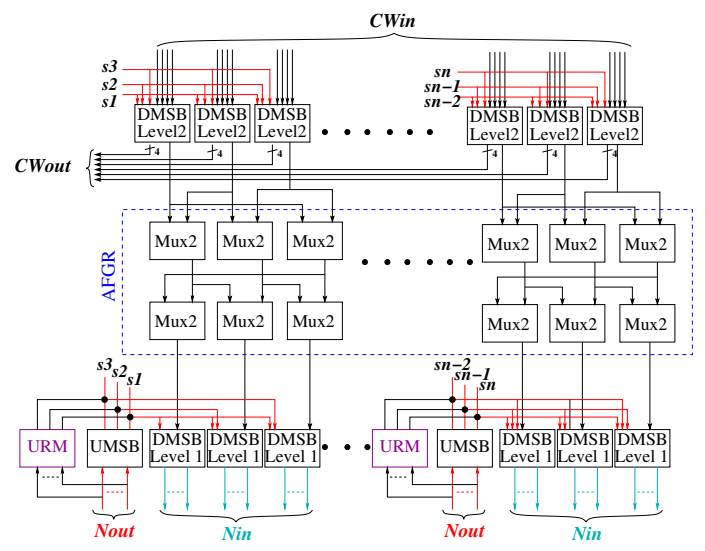

Fig. 4: Sbox with AFGR, DF and URM [17]

The technique called AFGR was proposed to reduce the impact of FGR and IFGR techniques on the FPGA area. This technique consists in removing one of the output multiplexer level to reduce the area overhead and makes the input multiplexer level common to all DMSBs (Fig.4 shows AFGR on DMSB1). With the technique called Distributed Feedbacks (DF), one or more feedbacks are connected to all DMSBs (as shown in Fig.4 with red bold lines). The technique called Upward Redundant Multiplexers (URM) permits to increase the defect tolerance of UMSBs by adding a multiplexer in parallel to the UMSB (illustrated in Fig.4).

\section{CRITICALITY}

Our aim is to measure the impact of each aforementioned technique on the criticality of the different blocks inside the FPGA.

\section{A. Definition}

The FPGA can be modeled using a graph $G=(N, A)$ such that :

1) all multiplexers inside the clusters and Sboxs represent the set of nodes $N$ of the graph G.

2) all signals connecting multiplexers represent the set of $\operatorname{arcs} A$ such as $(x, y) \in A$.

Each cluster and Sbox can be modeled using sub-graphs for DMSBs and UMSBs (illustrated Fig.5 for the cluster). The representation is the same for the Sbox. The criticality $\mathcal{C}$ of each node inside the cluster depends on the number of CLBs connected to this node compared to the number of existing paths to through it. 


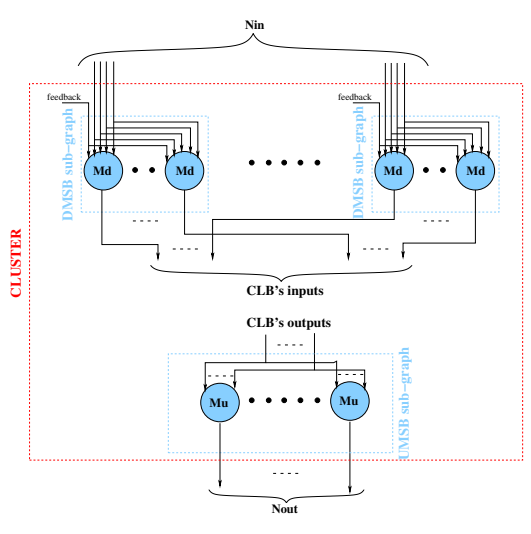

Fig. 5: Cluster's nodes

We denote $C \subset N$ the set of multiplexers inside the cluster and $D_{c} \subset C$ (resp $U_{c} \subset C$ ) the set of multiplexers inside DMSB (resp. UMSB). Then we have :

$$
\begin{aligned}
& \forall x \in D_{c}, \quad \mathcal{C}(x)=\frac{1}{d_{+}(x) * d_{-}(x)} \\
& \forall x \in U_{c}, \quad \mathcal{C}(x)=\frac{N_{c l b}}{d_{+}(x) * d_{-}(x)}
\end{aligned}
$$

where $N_{c l b}$ represents the number of CLBs per cluster, $d_{+}$the number of outputs of this node and $d_{-}$the number of inputs. In a cluster of a Mesh of Clusters FPGA, we have :

$\forall x \in D_{c}$ and $\forall y \in U_{c}, d_{-}(x)>d_{-}(y), N_{i n}>N_{c l b}$, then $\mathcal{C}(x)<\mathcal{C}(y)$.

Similarly, the criticality of each node inside the Sbox depends on the number of inputs/outputs per Sbox compared to the number of existing paths to through it. We denote $S \subset N$ the set of multiplexers inside the Sbox and $D 1_{s} \subset S$ (resp. $D 2_{s} \subset S$ and $U_{s} \subset S$ ) the set of multiplexers inside the MSBs (resp. DMSB1, DMSB2 and UMSB2). Then we have :

$$
\begin{aligned}
& \forall x \in D 2_{s}, \quad \mathcal{C}(x)=\frac{N_{\text {out }} / C W_{\text {in }}}{d_{+}(x) * d_{-}(x)} \\
& \forall x \in D 1_{s}, \quad \mathcal{C}(x)=\frac{N_{\text {out }} / N_{\text {in }}}{d_{+}(x) * d_{-}(x)} \\
& \forall x \in U_{s}, \quad \mathcal{C}(x)=\frac{N_{\text {in }}-N_{\text {out }}}{d_{+}(x) * d_{-}(x)}
\end{aligned}
$$

where $N_{\text {in }}$ and $N_{\text {out }}$ represents the number of inputs/outputs per cluster and $C W_{i n}$ the channel width. In a Sbox of a Mesh of Clusters FPGA, we have :

$\forall x \in D_{s}$ and $\forall y \in U_{s}, N_{\text {in }}-N_{\text {out }}>N_{\text {out }} / N_{\text {in }}$, $N_{\text {in }}-N_{\text {out }}>N_{\text {out }} / N_{C W \text { in }}, d_{+}(x) * d_{-}(x)>d_{+}(y) * d_{-}(y)$, then $\mathcal{C}(x)<\mathcal{C}(y)$.

We can conclude that multiplexers in UMSB are more critical than in DMSB.

\section{B. Impact of redundancy on criticality}

1) Classic Fine Grain Redundancy (FGR): With FGR (Fig.6a), the number of outputs per nodes inside DMSB and UMSB increases. In fact, each node can connect all inputs to 3 times more outputs. Then we have :

$$
\forall x \in N, \quad \mathcal{C}_{F G R}(x)=\frac{\mathcal{C}(x)}{3}
$$

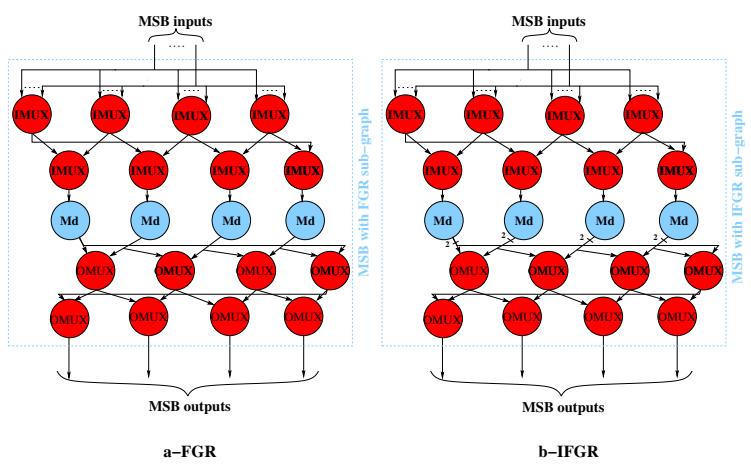

Fig. 6: MSB with FGR and IFGR

2) Improved Fine Grain Redundancy (IFGR): To adapt this technique to the Sbox and cluster architecture, the multiplexer's outputs inside each MSB are doubled (illustrated in Fig.6b). If the number of outputs per node inside MSBs is doubled, the criticality of each node is divided by 2 compared to FGR.

$$
\forall x \in N, \quad \mathcal{C}_{I F G R}(x)=\frac{\mathcal{C}_{F G R}(x)}{2}=\frac{\mathcal{C}(x)}{6}
$$

3) Adapted Fine Grain Redundancy (AFGR): With AFGR, the number of inputs per node inside MSBs increases (Fig.7). In fact, each node can use 2 extra inputs from its neighbor. The criticality of each node with AFGR becomes :

$$
\forall x \in N, \quad \mathcal{C}_{A F G R}(x)=\frac{\mathcal{C}(x)}{\left(d_{-}(x)+2\right) / d_{-}(x)}
$$

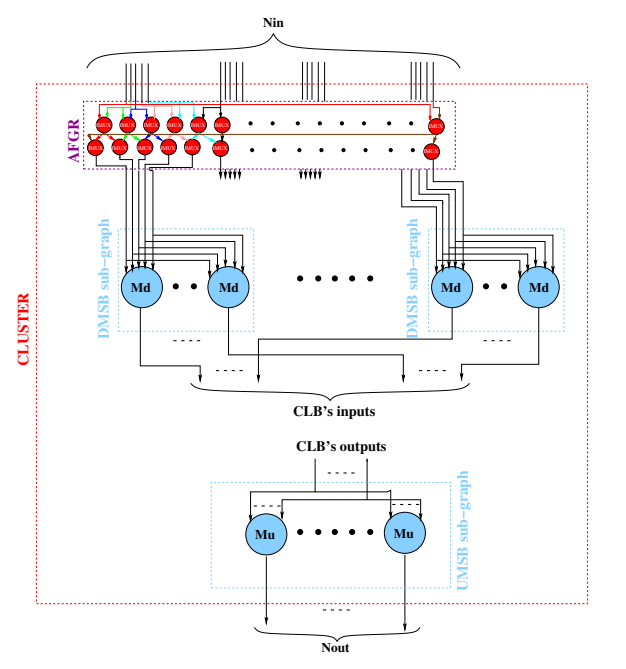

Fig. 7: Cluster with AFGR on dmsb 


\begin{tabular}{|c||c|c|c|c|c|}
\hline \multirow{2}{*}{$\begin{array}{c}\text { Hardware redundancy } \\
\text { technique }\end{array}$} & \multicolumn{3}{|c|}{ Sbox criticality } & \multicolumn{2}{c|}{ Cluster criticality } \\
\cline { 2 - 6 } & $\mathrm{Mu}$ & $\mathrm{Md} 1$ & $\mathrm{Md} 2$ & $\mathrm{Mu}$ & $\mathrm{Md}$ \\
\hline Without redundancy & $1(\mathrm{ref})$ & $0.086(\mathrm{ref})$ & $0.033(\mathrm{ref})$ & $1(\mathrm{ref})$ & $0.1(\mathrm{ref})$ \\
\hline FGR & $0.33(-67 \%)$ & $0.028(-67 \%)$ & $0.011(-67 \%)$ & $0.33(-67 \%)$ & $0.033(-67 \%)$ \\
\hline IFGR & $0.16(-84 \%)$ & $0.014(-84 \%)$ & $0.0053(-84 \%)$ & $0.16(-84 \%)$ & $0.016(-84 \%)$ \\
\hline AFGR & $0.83(-17 \%)$ & $0.071(-17 \%)$ & $0.027(-17 \%)$ & $0.83(-17 \%)$ & $0.083(-17 \%)$ \\
\hline DF & $1(-0 \%)$ & $0.046(-47 \%)$ & $0.017(-47 \%)$ & $1(-0 \%)$ & $0.053(-47 \%)$ \\
\hline URM & $0.01(-90 \%)$ & $0.086(-0 \%)$ & $0.033(-0 \%)$ & $0.01(-90 \%)$ & $0.1(-0 \%)$ \\
\hline
\end{tabular}

TABLE I: Impact on criticality

4) Distributed Feedbacks (DF): With this technique, the number of inputs per DMSB and per node inside DMSBs increases (Fig.8). The criticality of each node depends on the number of Distributed Feedbacks added $\left(N_{d f}\right)$.

$$
\forall x \in D_{c} \cup D 1_{s} \cup D 2_{s}, \mathcal{C}_{D F}(x)=\frac{\mathcal{C}(x)}{\left(d_{-}(x)+N_{d f}\right) / d_{-}(x)}
$$

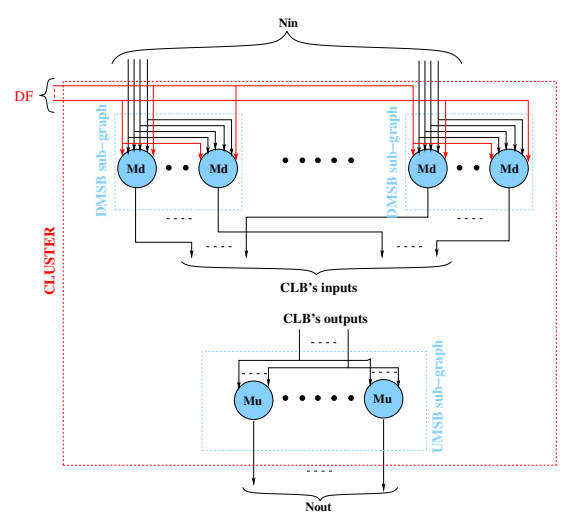

Fig. 8: Cluster with DF

5) Upward Redundant Multiplexer (URM): URM technique is applied only on the UMSB and has an impact on the criticality of each node inside UMSB (Fig.9). The criticality of each node depends on the number of URM $\left(N_{u r m}\right)$ added to the UMSB architecture.

$$
\forall x \in U_{c} \cup U_{s}, \quad \mathcal{C}_{U R M}(x)=\frac{\mathcal{C}(x)}{1+N_{U R M}}
$$

\section{Conclusion}

In this section, the impact of each technique on the criticality is calculated for a FPGA with a channel width $C W$ in $=C W$ out $=36$, a cluster with $N_{c l b}=10, N_{\text {in }}=24$, $N_{\text {out }}=12$ and 4 inputs per CLB. Table I presents the criticality value using all aforementioned equation depending on the considering node and redundancy technique. In this table, a 1 represents a highly critical block and a 0 a not critical block. Table I shows that without redundancy the most critical blocks are the multiplexers inside UMSBs, then

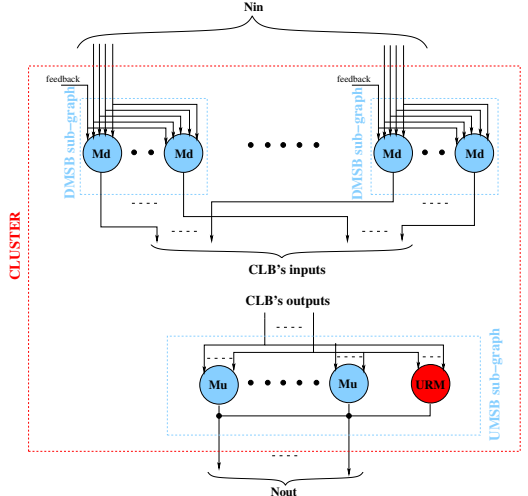

Fig. 9: Cluster with URM

the multiplexers inside the cluster's DMSBs. Finally, IFGR technique has the best impact on the DMSB multiplexer criticality (-84\%) and URM on the UMSB multiplexer (-90\%).

\section{EXPERIMENTAL EVALUATIONS}

In this section, our objective consists in evaluating the impact of different Local Redundancy Strategy (LRS) on the defect tolerance, area and timing of the FPGA depending on the criticality of the elements of the FPGA. Table I shows that multiplexers inside cluster's DMSB and multiplexers inside UMSBs are the most critical elements inside a Mesh of Clusters FPGA. IFGR has the best impact on the criticality but the area highly increases [17]. To reduce the area overhead others techniques (FGR, DF and AFGR) with a lowest impact on criticality are used on DMSB. Table II shows 5 different strategies studied in the experiments.

\begin{tabular}{|c||c|c|c|c|c|}
\hline $\begin{array}{c}\text { Local } \\
\text { Redundancy } \\
\text { Strategies }\end{array}$ & FGR & IFGR & AFGR & DF & URM \\
\hline LRS1 & & & & $\mathrm{X}$ & $\mathrm{X}$ \\
\hline LRS2 & & & $\mathrm{X}$ & & $\mathrm{X}$ \\
\hline LRS3 & & $\mathrm{X}$ & $\mathrm{X}$ & & \\
\hline LRS4 & & $\mathrm{X}$ & & $\mathrm{X}$ & \\
\hline LRS5 & $\mathrm{X}$ & & & & $\mathrm{X}$ \\
\hline
\end{tabular}

TABLE II: Local Redundancy Strategies 


\begin{tabular}{|c||c|c|c|c|}
\hline \multirow{2}{*}{\begin{tabular}{c}
\multirow{2}{*}{$\begin{array}{c}\text { Hardware } \\
\text { redundancy } \\
\text { technique }\end{array}$} \\
\cline { 2 - 3 }
\end{tabular}} & Mumber of defective multiplexer bypassed in the & \multirow{2}{*}{$\begin{array}{c}\text { FPGA area } \\
\text { overhead }\end{array}$} & $\begin{array}{c}\text { Avg Critical Path } \\
\text { Delay overhead }\end{array}$ \\
\hline FGR & $44(4.24 \%)$ & $502(48.36 \%)$ & $+64.6 \%$ & $+29.7 \%$ \\
\hline IFGR & $/$ & $878(84.58 \%)$ & $+150 \%$ & $+29.7 \%$ \\
\hline AFGR & $41(7 \%)$ & $502(48.36 \%)$ & $+30 \%$ & $+7.46 \%$ \\
\hline DF & $24(4 \%)$ & $600(57.8 \%)$ & $+112 \%$ & $+10 \%$ \\
\hline URM & $9(1.5 \%)$ & $180(17.34 \%)$ & $+17.34 \%$ & $+1 \%$ \\
\hline LRS1 & $12(1.15 \%)$ & $492(47.39 \%)$ & $+52.34 \%$ & $+7.81 \%$ \\
\hline LRS2 & $12(1.15 \%)$ & $394(37.95 \%)$ & $+21.84 \%$ & $+9.65 \%$ \\
\hline LRS3 & $180(17.34 \%)$ & $394(37.95 \%)$ & $+20.9 \%$ & $+22.36 \%$ \\
\hline LRS4 & $180(17.34 \%)$ & $492(47.39 \%)$ & $+51.4 \%$ & $+6.16 \%$ \\
\hline LRS5 & $12(1.15 \%)$ & $394(37.95 \%)$ & $+31.34 \%$ & $+17.09 \%$ \\
\hline
\end{tabular}

TABLE III: Local redundancy techniques performance

\section{A. Methodology}

For each 20 MCNC benchmark [19], we determine the smallest architecture in terms of logic blocks number, to place and route all applications. For all simulations, this architecture correspond to a 36x36 FPGA with a channel width of 36, a cluster with 10 CLBs, 24 inputs (6 per crossbar down), 12 outputs. This cluster architecture correspond to the minimal architecture to place and route each benchmark with 10 CLBs per cluster. Then, defects are randomly injected on multiplexers in the FPGA interconnect to make the application not routable. A defect is modeled by undefined value (stuckopen) at the multiplexer output that makes the multiplexer unusable. This number of defective multiplexers corresponds to the defect tolerance of each technique. The number of mux2 added with each technique is compared to initial architecture to evaluate the area increase. Finally, the Critical Path Delay (CPD) of the application is determined for each technique and compared to the initial architecture. For all simulations, we considered that all clusters and Sbox inputs are faultfree. Configuration memory is protected against the defect by using error detection/correction codes [20] [21]. The design under study are the FPGA architecture enriched with all aforementioned strategies : LRS1, LRS2, LRS3, LRS4 and LRS5.

\section{B. Defect tolerance, area and timing results}

Table III presents the performance of the different techniques in term of tolerance, area and timing. DF, FGR, IFGR, AFGR and URM techniques add extra multiplexers inside all MSBs of the FPGA compared to Local Redundant Strategy (LRS). In fact, with LRS the redundancy is only applied on the most critical elements of the Sbox and cluster. Compared to hardware redundancy techniques, LRS reduces the defect tolerance $(-18.65 \% \mathrm{avg})$ but the area and the CPD are also reduced (resp $-52.58 \%$ and $-18 \%$ avg). We can show that LRS1 and LRS4 techniques permits to bypass a high number of mux2 $(47.39 \%)$ with a FPGA area overhead of about $+52 \%$.
On the contrary with LRS2, LRS3 and LRS5, the impact on the area overhead is reduced (resp. $+21.84 \%,+20.9 \%$ and $+31.34 \%$ ) with a less number of multiplexers bypassed (37.95\%). In conclusion, using local hardware redundancy on the most critical blocks inside the FPGA permits to tolerate lot of defects with an area overhead reduced compared to an hardware redundancy applied on all the FPGA. For the Critical Path Delay, LRS1 is the best solution $(-7.81 \%)$ because new paths are added to the cluster that replace defective elements to limit the impact on the Critical Path Delay. Finally, the best trade off is for LRS2 with $37.95 \%$ of multiplexers bypassed, an area overhead of $+21.84 \%$ and an increase of Critical Path Delay of $+9.65 \%$.

\section{Mesh vs Mesh of Clusters Architecture}

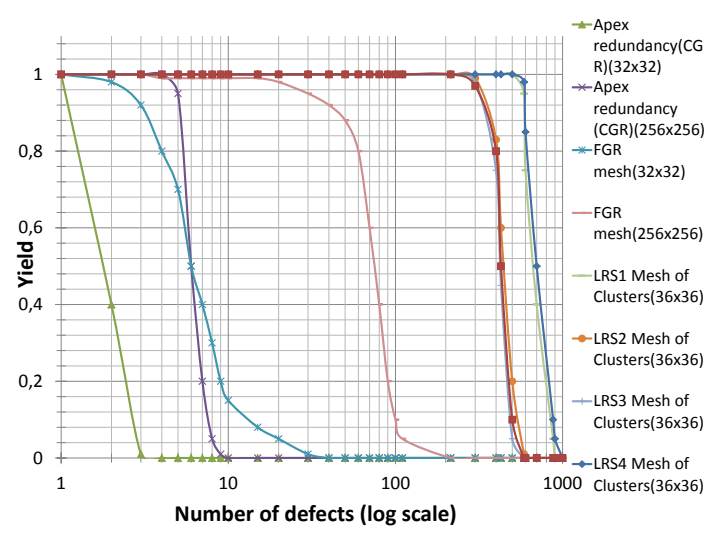

Fig. 10: Impact on FPGA yield

In this paragraph, we compared the FPGA yield to the number of defects (Fig.10) and timing (Fig.11) for industrial mesh FPGA [12] [13] with different redundancy techniques (FGR, IFGR) and all presented techniques on our Mesh of Clusters architecture using local redundancy strategies (LRS1, LRS2, LRS3, LRS4 and LRS5). The yield represents the 
FPGA functionality after manufacturing. It shows that all techniques using local redundancy applied on a Mesh of Clusters architecture permits to tolerate around 4 times more defects than FGR,IFGR, CGR applied on a mesh-based industrial FGPA interconnect [12] [13] [9]. We can also compare all techniques using local redundancy applied on Mesh of Clusters architecture. Best results are obtained using LRS1 and LRS4 with around 492 defects tolerated. Finally, LRS2, LRS3 and LRS5 have the same impact with 394 defects tolerated. Fig.11, shows the impact of each method on the average Critical Path Delay overhead when local hardware redundancy is applied. Each proposed methods (LRS1, LRS2, LRS3, LRS4 and LRS5) reduce the CPD overhead compared to FGR and IFGR because, the hardware redundancy is not applied on the total FPGA architecture to limit the area overhead. As mentioned before LRS1 is the best solution (-7.81\%). Finally, LRS permits the designer to fix a trade off between area, defect tolerance or delay.

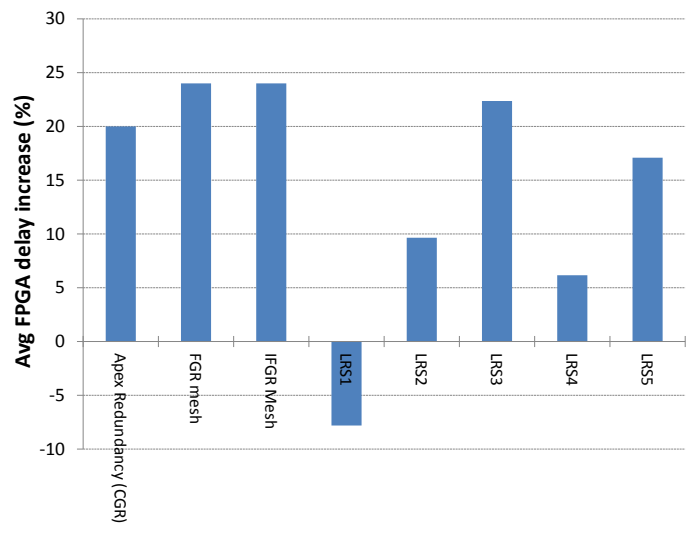

Fig. 11: Impact on Critical Path Delay

\section{CONCLUSION AND FUTURE WORK}

In this paper, we determined the most critical blocks inside the FPGA to applied different local hardware redundancy strategies to the Mesh of Clusters topology. It permits to tolerate 4 times more defects than a classic hardware redundancy applied in a mesh architecture. Each method uses hardware redundancy in the FPGA local interconnect. However our investigation indicates that the choice of defect tolerant techniques has a significant impact on area and critical path delay. With these strategies, we obtain the best trade off between the number of defects bypassed (37.95\%), the FPGA area overhead $(21.84 \%)$ and critical path delay $(9.65 \%)$. Furthermore, with our techniques the designer can choose his trade off between area, defect tolerance or delay. For future works, it will be interesting to study the impact of Coarse Grain Redundancy techniques.

\section{ACKNOWLEDGMENT}

This work is a part of the project Robust FPGA ANR 11 INS-02, funded by The French National Research Agency, The Pole Systematic and The Pole Minalogic.

\section{REFERENCES}

[1] Rahul Jain, Anindita Mukherjee, and Kolin Paul. Defect-aware design paradigm for reconfigurable architectures. In IEEE Computer Society Annual Symposium on Emerging VLSI Technologies and Architectures, pages 6-pp. IEEE, 2006

[2] Honey Goel and Daren Dance. Yield enhancement challenges for 90 $\mathrm{nm}$ and beyond. In Advanced Semiconductor Manufacturing Conference and Workshop, 2003 IEEEI/SEMI, pages 262-265. IEEE, 2003.

[3] Helia Naeimi and André DeHon. A greedy algorithm for tolerating defective crosspoints in nanopla design". In International Conference on Field-Programmable Technology, pages 49-56. IEEE, 2004.

[4] Nicola Campregher, Peter YK Cheung, George A Constantinides, and Milan Vasilko. Analysis of yield loss due to random photolithographic defects in the interconnect structure of fpgas. In Proceedings of the 2005 ACM/SIGDA 13th international symposium on Field-programmable gate arrays, pages 138-148. ACM, 2005.

[5] Vijay Lakamraju and Russell Tessier. Tolerating operational faults in cluster-based fpgas. In Proceedings of the 2000 ACM/SIGDA eighth international symposium on Field programmable gate arrays, pages 187-194. ACM, 2000.

[6] Abderrahim Doumar, Satoshi Kaneko, and Hideo Ito. Defect and fault tolerance fpgas by shifting the configuration data. In International Symposium on Defect and Fault Tolerance in VLSI Systems. DFT'99, pages 377-385. IEEE, 1999.

[7] San Jose CA Xilinx. Easypath solutions. In http://www.xilinx.com/ products/easypath/, 2005.

[8] Raphael Rubin and André DeHon. Choose-your-own-adventure routing: lightweight load-time defect avoidance. ACM Transactions on Reconfigurable Technology and Systems (TRETS), 4(4):33, 2011.

[9] Altera Corporation. Apex redundancy. In http://www.altera.com/products /devices/apex/features/apx-redundancy, 2005.

[10] John D. Corbett Xilinx. The xilinx isolation design flow for fault-tolerant systems. In http://www.xilinx.com/support/documentation/white_papers Iwp412_IDF_for_Fault_Tolerant, 2012.

[11] A. Ben Dhia, S. Ur Rehman, A. Blanchardon, L. Naviner, M. Benabdenbi, R. Chotin-Avot, H. Mehrez, E. Amouri, and Z. Marrakchi. A defect-tolerant cluster in a mesh sram-based fpga. In in FPT, Kyoto, Japan, pp. 434-437 (2013).

[12] AJ Yu and Guy G Lemieux. Fpga defect tolerance: Impact of granularity. In International Conference on Field-Programmable Technology, pages 189-196. IEEE, 2005.

[13] AJ Yu and Guy GF Lemieux. Defect-tolerant fpga switch block and connection block with fine-grain redundancy for yield enhancement. In International Conference on Field Programmable Logic and Applications, pages 255-262. IEEE, 2005.

[14] E. Amouri, A. Blanchardon, R. Chotin-Avot, H. Mehrez, and Z. Marrakchi. Efficient multilevel interconnect topology for cluster-based mesh fpga architecture. In International Conference on ReConFigurable Computing and FPGAs, 2013.

[15] Andre DeHon. Reconfigurable architectures for general-purpose computing. 1996.

[16] Joachim Pistorius and Mike Hutton. Placement rent exponent calculation methods, temporal behaviour and fpga architecture evaluation. In Proceedings of the 2003 international workshop on System-level interconnect prediction, pages 31-38. ACM, 2003.

[17] A. Blanchardon, R. Chotin-Avot, H. Mehrez, and E. Amouri. Improve defect tolerance in a cluster of a sram-based mesh of cluster fpga using hardware redundancy. In in FPL, Munich, Germany, 201.

[18] AJ Yu and Guy GF Lemieux. Defect-tolerant fpga switch block and connection block with fine-grain redundancy for yield enhancement. In International Conference on Field Programmable Logic and Applications, pages 255-262. IEEE, 2005.

[19] Lgsynth93 benchmark set: Version 4.0. In Technical report, Collaborative Benchmarking Laboratory, 1993.

[20] Charles W Slayman. Cache and memory error detection, correction, and reduction techniques for terrestrial servers and workstations. IEEE Transactions on Device and Materials Reliability, 5(3):397-404, 2005.

[21] Fabrice Monteiro, Stanislaw J Piestrak, Houssein Jaber, and Abbas Dandache. Fault-secure interface between fault-tolerant ram and transmission channel using systematic cyclic codes. In On-Line Testing Symposium, 2007. IOLTS 07. 13th IEEE International, pages 199-200. IEEE, 2007. 